\title{
El impacto del desarrollo de las tecnologías de la información y las comunicaciones y de la globalización económica en la enseñanza de las humanidades: diagnóstico sucinto y propuestas de cambio
}

\author{
" The Impact of the development of information and \\ communication technologies, and of globalization \\ in the teaching of the humanities: prognosis and \\ suggestions for change
}

\begin{tabular}{l}
\hline Priscilla Gac-Artigas \\
\hline Monmouth University \\
\hline Gustavo Gac-Artigas \\
\hline Academia Norteamericana de la Lengua Española (ANLE) \\
\hline
\end{tabular}

\section{Resumen}

En este artículo se presenta una sucinta descripción de las razones económicas e ideológicas determinantes del estado actual de la enseñanza de las humanidades en los Estados Unidos. Discutiremos cómo, por un lado, la concentración de poder del mundo editorial y la de la industria de las comunicaciones en un puñado de consorcios, y por el otro, la parcelación de la información en los medios y la manipulación de la tecnología, están afectando las capacidades de lectoescritura, pensamiento crítico y expresión del individuo. Finalmente, argumentaremos sobre la necesidad y la forma de transformar el enfoque de la enseñanza de la literatura y otras disciplinas humanísticas de cara a revertir esta crisis formando individuos que desafíen el statu quo de poder económico, ideológico y político.

Palabras clave: globalización, monopolio de la información, disciplinas humanísticas, pensamiento crítico, enseñanza de las humanidades.

\begin{abstract}
In this paper, will present a concise description of the economic and ideological reasons determining the current state of the teaching of the humanities. We will discuss how the concentration of power of the publishing world and that of the communications industry in a handful of consortia, on the one hand, and the parceling out of information in the media as well as the manipulation of technology on the other, is affecting people's literacy, critical thinking and communication skills. Finally, we will argue for the need, and the ways, to transform the approach of teaching literature and other humanistic disciplines in order to overturn this crisis by educating individuals who could challenge the status quo of economic, ideological and political power.
\end{abstract}

Keywords: globalization, information monopoly, humanities, critical thinking, the teaching of the humanities.

Cómo citar este artículo:

Gac-Artigas, P. \& Gac-Artigas, G. (2019). El impacto del desarrollo de las tecnologías de la información y las comunicaciones y de la globalización económica en la enseñanza de las humanidades: diagnóstico sucinto y propuestas de cambio. Revista Amauta, 17(34), pp.27-42. DOI: http://dx.doi.org/10.15648/am.34.2019.4
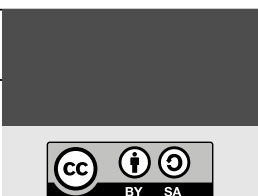

() 2019

Correspondencia de autor:

pgacarti@monmouth.edu ggacarti@gmail.com

\section{Recibido:}

$30 /$ Abril/2019

Aceptado:

2/Junio/2019

Publicado:

28/Junio/2019 


\section{Introducción}

\section{Hubo una época en que las humanidades fueron parte de la vida, y en su diferencia, se expresaban}

De viaje al VII Taller Internacional La enseñanza de las disciplinas humanísticas, en Cuba, la pregunta era, ¿para qué?, ¿a quién nos dirigimos? Soy escritor, y hombre de teatro, trabajo con la imagen y la palabra, muero en el escenario y renazco en la mente del espectador, muero en las páginas de un libro, en un poema, en busca de un lector.

El actor desaparece en el personaje, el personaje muere y renace en la mente del espectador, despojado de falsas vestimentas, vestido de nuevas experiencias, renaciendo al apagarse las luces. El texto literario se reescribe en cada lector y cada reescritura es diferente y se alimenta de otros amores, de otras decepciones, de otras lecturas, de nuevos pensamientos y escapa de las manos de aquellos que quieren encarcelarlo.

Nos encontramos en un mundo globalizado, un mundo en que todo está al alcance de nuestras manos, tan al alcance, que se vuelve inaccesible, un mundo de algoritmos que quieren regir nuestras sensaciones, nuestros gustos, un mundo no muy diferente de otros mundos. Mundos que quieren uniformar el pensamiento, que llaman a pensar lo permitido, a leer lo por otros decidido, a enterrar el pensamiento y las voces que se levantan como en aquella lejana época en que el teatro salió a las calles, los estudiantes abandonaron las estériles aulas, los escritores mojaron sus plumas en la realidad y la fantasía, en que el prohibido prohibir alimentaba las humanidades más allá de un congreso, más allá de repetidas citas. Un mundo donde las voces discordantes son silenciadas 0 ahogadas en el remolino de lo accesible.

Al abandonar este recinto, saldré a las calles a buscar la respuesta, esperando no encontrarla para poder seguir preguntando y preguntándome.

En mayo de 2019, Naciones Unidas hizo sonar una campanada de alerta, la humanidad está en riesgo por la pérdida de la biodiversidad. Un millón de especies, de los ocho millones existentes, están en peligro de extinción. Lo interesante es que no tenemos que hacer nada para llegar a la destrucción, basta que dejemos las cosas como están 
y no tomemos medidas. Pero lo cierto es que siempre el tomar medidas implica cambio, y a veces es más fácil no cambiar nada y vivir tranquilos.

Y qué tiene que ver esto con las humanidades, me pregunté, pensando quizás que entre los bichos raros a desparecer, están los escritores, los filósofos, raros seres que terminaban sus vidas en el ostracismo o bebiendo cicuta; pensaba en los hombres y mujeres de teatro que tiemblan pensando en futuras obras escritas, financiadas y difundidas por Netflix, como ya lo hace con el cine, en esos cantos de sirena a los que el creador, o el crítico cede.

\section{¿Cómo no ceder si ellos manejan nuestro público?}

El mundo editorial y su concentración está, a mi entender, caminando a la destrucción tanto del lector como del escritor. En el caso del lector, poco a poco están decidiendo por nosotros, qué leer, en el caso del escritor; qué publicar, aquello que llegará a las manos de la masa. El algoritmo que decide qué debo leer está llevando gradualmente a uniformar el pensamiento, nos sentiremos halagados al leer, lo que es hot, y cada mañana, a primera hora, me llegarán las noticias del día junto al nombre del libro hot de la semana, gentileza de Google, Apple, CNN y el New York Times. Y el libro será igual que una humilde polera que dejó de ser polera para transformarse en cartel de propaganda de una marca, que ocupa el pecho y la espalda del orgulloso portador de la prenda. No interesa que sea de algodón o poliéster, interesa la marca y que se vea que yo también soy hot y soy normal y me gusta lo que es el gusto de todos. $Y$ hoy en mi polera, ni siquiera es la 's' de Superman la que ocupa el primer plano, ese al menos es un personaje. No, su lugar es ocupado por una marca, lo personalizado es peligroso. $Y$ veremos surcar por nuestros cielos, no una nave, o un ave, o un meteorito, sino la P de Planeta, la RH de Random House, o, imi sueño! adelantándome al futuro, un pulgar para arriba.

Al escritor, por lo que interesa la cartera de autores de las pequeñas editoriales que desaparecen en las fauces de los monstruos, le será sugerido sobre qué escribir, le soplarán al oído lo que necesita hacer para brillar en el firmamento literario, el qué piden los comerciantes y los censores, dado que entre ellos no hay gran diferencia, el qué se necesita para que, ligero como un paquetito de palomitas de maíz, o pesado como un ladrillo de muros del pasado, flote en las manos delicadas de un lector que 
no quiere fatigar su mente con obras que le exijan un mínimo esfuerzo. Por favor, un segundo de pensamiento crítico, ruega el escritor relegado a desaparecer en este tsunami editorial, tsunami que inunda y se pasea por las aulas de las universidades arrasando a su paso con los ramos humanísticos, para uniformar el pensamiento del futuro consumidor en esta sociedad digital que aísla y encajona para eliminar toda posibilidad de pensamiento inquisitivo y mantener el poder.

Sin el desarrollo de la humanidad, sin la riqueza que aportan el aprender a disfrutar de la creación artística, el cabalgar y soñar por los caminos de La Mancha o de Macondo, el escuchar atentos las voces de la historia, el sumergirse en el inconstante río heracliano, el practicar una amplia solidaridad, no lograremos enfrentar al cíclope de la lectura uniformada que está desarrollando ese pensamiento uniformado. Y si no tomamos medidas, el mundo, por la falta de diversidad en el pensamiento, marchará a su destrucción, como dice el informe de Naciones Unidas.

\section{Desarrollo}

\section{Hacia una nueva aproximación a la enseñanza de las humanidades}

Queda claro que en una situación como la descrita donde se nos está llevando a la destrucción de aquello que nos distingue de los otros seres, como educadores universitarios (y preferimos este término al de profesor por sus profundas implicaciones), debemos tomar conciencia del importante rol que estamos llamados a jugar para subvertirla. Decía Paulo Freire (2008) en sus Cartas a quien pretende enseñar, que el educador debería estar "permanentemente disponible para repensar lo pensado, para revisar sus posiciones" (p.45). En ese sentido nuestras metodologías de enseñanza deben establecer prioridades y la primera, a nuestro entender, es establecer ¿para qué enseñamos? antes de decidir el ¿qué enseñar? y el ¿cómo enseñarlo? ¿Queremos simplemente enseñarles en forma unidireccional las destrezas y entregarles las herramientas para garantizarles el poder desempeñar un trabajo en el futuro, como se estila cada vez más, o, por el contrario, queremos educarlos en un sistema caracterizado por la retroalimentación multidireccional y la crítica para crear un ciudadano informado, capaz de participar activamente de la vida en sociedad? 
Nosotros hemos optado por lo segundo, y por años hemos articulado enseñanza y aprendizaje con el fin de poner a la disposición de los alumnos las herramientas necesarias para aprender a leer de forma crítica de modo que lleguen a comprender lo leído, a objetivar, estudiar y comprender la realidad que los rodea y a comunicar en forma creativa el resultado de esos encuentros. El objetivo ha sido doble: enseñarlos a enseñar, pero también enseñarlos a aprender'.

Como en toda transformación de las metodologías de enseñanza, el camino no es recto ni es fácil transitarlo y como decía Freire, debemos estar abiertos a aprender de las experiencias y modificar nuestras posiciones. En esta sección nos centraremos en posibles medidas que pueden ayudarnos a sortear los obstáculos que nos permitan cambiar el rumbo de la educación. Resumiremos los obstáculos en tres para de ahí, partir compartir soluciones que han probado ser efectivas en el aula.

El primer obstáculo a sortear, es la falta de preparación y motivación con la que llegan los estudiantes a la universidad. Sin afrontar esta carencia, será imposible envolver a los alumnos en el proceso. El segundo, es el del estereotipo establecido de la superioridad de las disciplinas científicas y tecnológicas sobre las humanidades y las Ciencias Sociales, que también se traduce en los salarios percibidos por quienes obtienen diplomas en estas carreras. Y finalmente, la marginalización y la imposición tácita de la regla del silencio.

1 Nuestro trabajo de investigación y aplicación práctica en el aula puede ser resumido en las siguientes etapas: Primera etapa: Publicación de dos gramáticas: Directo al grano, A Complete Reference Manual for Spanish Grammar (1999) y Sans détour, A Complete Reference Manual for French Grammar (1999) cuyo objetivo era presentar los paradigmas gramaticales lo más cercano posible a fórmulas matemáticas para estudiantes de segunda lengua.

Segunda etapa: iA la perfección! Para dominar la mecánica de la escritura (2009). "metodología integral de enseñanza-aprendizaje multidireccional con retroalimentación y medición continua", cuyo objetivo fue subvertir la estructura jerárquica vertical tradicional del aula para convertir al alumno en protagonista de su propio aprendizaje.

"iA la perfección! Para dominar la mecánica de la escritura: una nueva aproximación a la enseñanza de la escritura en español". La investigación en didáctica de la lengua y la literatura: situación actual y perspectivas de futuro, CD-ROM. Coords. María Pilar Núñez Delgado and José Rienda. Sociedad Española de Didáctica de la Lengua y la Literatura, Universidad de Granada, 2011: 611-628.

Tercera etapa: EssayGPS/EnsayoGPS, aplicación de escritura cuyo objetivo es contribuir al desarrollo del pensamiento crítico a través del fortalecimiento de las destrezas de escritura y comunicación, funcionando como un GPS. Gracias a su utilización constante el usuario va automáticamente internalizando los patrones para la redacción coherente, organizada y estructurada de diferentes tipos de ensayos, lo que les permite concentrarse y profundizar en el contenido.

Cuarta etapa: "Escribir para el cambio" que engloba todas las etapas anteriores y donde se añade el énfasis en la creatividad para fortalecer las competencias de lectoescritura y de razonamiento crítico así como la autoestima de los alumnos, esencial para su proactividad como agente de cambio y su liderazgo dentro de la sociedad (Nathan, 2018). 
Desde hace algún tiempo, nuestra investigación ha estado articulada en el desarrollo del pensamiento crítico y el fortalecimiento de las destrezas de comunicación para llevar a los estudiantes a tomar la iniciativa de participar activamente en su propio proceso de aprendizaje y a convertirse en miembros activos en el aula y, por extensión en la sociedad. Hace un par de años añadimos a los objetivos de base el desarrollo de las competencias creativas del individuo. Estudios recientes (Church, Morrison, y Ritchhart, 2011) confirman que la creatividad no solo fortalece las competencias de lectoescritura y de razonamiento crítico, sino la autoestima de los alumnos, esencial para su proactividad como agente de cambio y su liderazgo dentro de la sociedad (Nathan, 2018).

En una sociedad donde la enseñanza de las humanidades con las competencias de pensamiento, análisis, retórica y creatividad que estas le aportan al individuo está en peligro; sociedad donde se intenta encajonar el pensamiento por medio de algoritmos, se necesita más que nunca revertir el sistema educativo. Se necesita enfatizar la relevancia de individuos capaces de observar el mundo con ojos inquisitivos y críticos; individuos con la confianza y las competencias necesarias para adaptarse rápidamente y de manera innovadora a los vertiginosos cambios producidos por el desarrollo tecnológico; individuos cuya imaginación esté abierta y capacitada tanto para la investigación científica y el desarrollo de la técnica, como para la creación estética y literaria, o la reflexión filosófica; individuos que sean miembros activos de la sociedad, con el arrojo para alejarse de los caminos más transitados y abrir nuevos rumbos.

El desafío ha sido, entonces, el de reforzar las destrezas de lectoescritura de alumnos acostumbrados a un mínimo nivel de lectura, de análisis y redacción a través del desarrollo de sus capacidades creativas. Un individuo que es capaz de entender lo que lee, es también capaz de analizar, sintetizar la información, relacionarla con su entorno y producir nuevo conocimiento. La metodología puede ser aplicada a cualquier curso que se enseñe. Las experiencias que describiré provienen de la enseñanza de cursos avanzados de redacción o de literatura hispánica. En ambos casos, se partió de la premisa de la necesidad de romper con los tres obstáculos mencionados: las carencias de formación con que llegan los alumnos; su desconocimiento y falta de aprecio de las materias en que pueden obtener las herramientas para superar esas carencias; y el silencio que se autoimponen como consecuencia de la falta de conciencia de que su falta de preparación se debe más a fallas del sistema que a situaciones individuales y piensan, "no sé" por ende, mejor dejo que 
hablen los que saben². El rol del educador es romper con ese ciclo y despertar en los alumnos el placer por la palabra escrita, su lectura, su análisis y su producción. ¿Cómo? Poniendo a su disposición las herramientas para aprender a leer, pensar, escribir y expresarse críticamente, y en el proceso, aprender a aprender.

\section{Primera etapa: reaprender a leer y a pensar}

En general, en el ámbito universitario, se habla de las carencias de escritura y ya muchas universidades han creado centros para ayudar a los estudiantes a mejorar su redacción académica. Sin embargo, se da por sentado que todos los alumnos llegan a la universidad sabiendo leer, y poco se habla y se hace por remediar las evidentes carencias de lectura cuando de todos es sabido la estrecha correlación existente entre lectura y escritura: los buenos escritores son siempre buenos lectores; quien no lee exhibe un léxico pobre y una ortografía ídem. La realidad que hemos constatado es que los alumnos saben juntar las letras, pero son muy pocos los que son capaces de entender lo que se esconde entre las líneas, y más allá de las líneas lo que nos está llevando a esa sociedad no pensante de la que hablamos al comienzo.

¿Cómo resolver las carencias de lectoescritura y análisis? Partiendo de la premisa de ¿para qué enseñamos?, buscamos ¿qué enseñar? y ¿cómo enseñarlo? para lograr nuestros objetivos. En la primera sesión de clase, comenzamos por discutir con los estudiantes qué significa leer críticamente, y ello lo ejemplificamos partiendo de las etapas para aprender a leer de manera crítica descritas por Church, Morrison y Ritchhart en su libro Making Thinking Visible (2011).

1. Observar detenidamente y poder describir con detalles lo que se observa.

2. Cuestionar y cuestionarse sobre el objeto de observación.

3. Imaginar posibles explicaciones o interpretaciones.

4. Considerar diferentes puntos de vista o perspectivas.

5. Hacer conexiones.

6. Razonar a partir de evidencias y llegar a conclusiones.

2 De acuerdo al informe "Born to Win, Schooled to Lose: Why Equally Talented Students Don't Get Equal Chances to Be All They Can Be," difundido por el Georgetown University Center on Education and the Workforce en mayo del 2019 en EE. UU. un niño del cuartil socioeconómico más bajo, con puntajes altos de exámenes en Kindergart en tiene solo tres en diez probabilidades de tener una educación universitaria y un buen trabajo al comienzo de su carrera, en comparación con las siete de diez probabilidades para un niño en el cuartil superior con puntaje bajo en las pruebas. 
Luego, hicimos como en el teatro: ejercicios de calentamiento para entrenar al cerebro utilizando estas estrategias una por una a partir del estudio de una cita breve, relevante a los temas de la literatura, la importancia de la lectura crítica, del estudio, de la reflexión, de la expresión oral y escrita, la conexión literatura/arte/ artista y sociedad, etc.

El objetivo de que fuera una cita breve era el de facilitar el trabajo de reflexión, evitar la dispersión y estimular al alumno a escudriñar la frase hasta lograr extraer de esta el máximo de significado posible, a pensar críticamente sobre cada detalle, a buscar en el diccionario o en la enciclopedia cualquier palabra o alusión desconocida que le impidiera entender la frase en todas sus capas. En el nombre del autor de la cita se incluía un enlace a su biografía con el fin de provocar la curiosidad intelectual para conocer más sobre el autor o sobre el tema, a establecer conexiones, a procesar y sintetizar las ideas y expresarlas por escrito, en preparación a la discusión en grupo que se daría en clase. Tres días antes de la clase siguiente, cada estudiante debía enviar por correo electrónico a la profesora y al grupo, su reflexión o preguntas que le provocara la cita, conexiones con otros textos estudiados, canciones u obras de arte por ellos conocidas, o referencias a los comentarios de sus pares. Dos estudiantes asignados por la profesora estaban encargados, en el lugar de ella, de dirigir la discusión sobre la cita que abriría la clase.

Como ejemplo, en la primera sesión utilizamos "Lee y conducirás, no leas y serás conducido", Santa Teresa de Jesús (1515-1582). Por razones evidentes, en la primera sesión, esta actividad se realizó completamente en clase para mostrar el camino.

A continuación, algunos de los comentarios más elocuentes expresados por los alumnos en la autoevaluación de este tipo de ejercicio al final del semestre: "el ejercicio sobre la cita me ayudó a desarrollar mis destrezas de pensamiento crítico al permitirme ver la cita desde perspectivas diferentes"; "pude aplicar cada cita no solamente a la literatura, sino a diferentes aspectos de la vida"; "el ejercicio me ayudó a profundizar en el sentido de la cita hasta ser capaz de desmenuzarla palabra por palabra para lograr entenderla en su significado mayor"; "la posibilidad de pensar de manera individual en nuestro propio tiempo y luego compartir lo que pensábamos por mail me permitió contrastar mis opiniones con las de mis compañeros y expandir mi entendimiento de la cita antes de llegar a la discusión en clase donde se fomentaba 
un análisis cada vez más profundo de otros textos"; "personalmente disfruté mucho de este ejercicio y hubiera deseado haber tenido esta experiencia en todas mis otras clases"; "lo que más me gustó del ejercicio fue darme cuenta de cómo unas pocas palabras pueden tener tanto significado y ser interpretadas en formas diferentes y aplicadas a contextos diferentes dependiendo del bagaje cultural y las experiencias de cada uno".

\section{Segunda etapa: más allá de las palabras}

¿Cómo romper la idea predominante en la era digital de la superioridad de las disciplinas Científicas y Tecnológicas sobre las Humanidades y las Ciencias Sociales? Convenciendo a los alumnos de que las Ciencias y la Tecnología no son superiores a las Ciencias Humanas; que estas últimas les ayudan a desarrollar el pensamiento crítico, la creatividad y la capacidad de comunicar a otros sus propuestas, para convertirse en quien aporta innovación, en quien cuestiona la sociedad para lograr el bienestar de todos, y no se limita a pulsar un botón y reproducir el espíritu de borrego.

Para ello, se debe proceder a una lectura, análisis, discusión y proyección de los textos literarios o artísticos estudiados en tres etapas: las líneas, muy importante cuando se enseña a estudiantes de español como segunda lengua, nuestro caso, pero también, como estrategia de nivelación; entre las líneas, para entender el mensaje o los símbolos tras las palabras; más allá de las líneas, para expresar creativamente la interpretación personal del mismo. Los seis pasos para leer de manera crítica descritos anteriormente son utilizados sistemáticamente en esta etapa para interiorizar el camino del pensamiento reflexivo en el alumno y que pueda reproducir esta forma de pensar, analizar, organizar, sintetizar, conectar, crear y proponer en cualquier contexto.

Los textos a estudiar pueden ser escogidos por temas, autor, género literario, entre otras razones. En nuestro caso, en el curso de redacción, trabajamos por temas relevantes a los alumnos y a la sociedad en que vivimos: distintas caras de la discriminación, la migración, el rol del artista en la sociedad, la educación, etc. Para el curso de introducción a la literatura hispánica trabajamos con obras de diversos géneros: poesía, cuento, drama y ensayo, y de diferentes épocas, pero todos, textos relativamente breves. Al finalizar el semestre, el consenso fue el siguiente: 
Cuando comenzó esta clase, me preocupaba mi capacidad para comprender y analizar poesías y cuentos en español. El primer día luché mucho con "Las medias rojas" ${ }^{\prime \prime}$, pero ahora es uno de mis cuentos favoritos. Siento que a través del estudio exhaustivo de diferentes géneros literarios, y de la posibilidad de establecer conexiones con las obras de arte estudiadas en clase y en las exposiciones visitadas, y con la música, logré no solo entender mejor la literatura hispana sino también la inglesa. Espero poder usar lo que aprendí en esta clase para reflexionar e interpretar las lecturas en todas mis otras clases. (S. S. primavera, 2019)

\section{Tercera etapa: escribir para cambiar}

¿Cómo mantener el necesario silencio reflexivo, pero al mismo tiempo fomentar la ruptura del silencio opresor (Freire, 2008) que predomina en la mayoría de los alumnos y se respira en la mayoría de las aulas? Llevando el resultado obtenido en las etapas anteriores de reflexión al próximo nivel, el de la creación. El entregarles las herramientas necesarias les permitió a los alumnos poder reflexionar, analizar e intercambiar sus ideas con sus pares, en pequeños grupos o como clase, les dio confianza en sus habilidades creativas y los hizo conscientes de su potencial para usar sus voces y sus palabras para ejercer su voz dentro del aula y la sociedad.

Todos los escritores estudiados en clase se han preocupado por lo que creían correcto y aquellos que leen su trabajo son cambiados por sus ideas. Espero que algún día mi capacidad de escritura sea tan poderosa como la de ellos y pueda cambiar a las personas con mi filosofía (S. S. primavera, 2019)

Dentro de esta nueva aproximación a la enseñanza, no solamente se fortalecieron las destrezas de lectoescritura y de reflexión y análisis, sino que a la par se fomentó el sentido de independencia, participación y creatividad, logrando en el proceso despertar el placer de la palabra expresado en una nueva idea, en un nuevo escrito. Pudimos observar que, a partir del análisis de textos literarios, pictóricos, o de la cultura popular (canciones), y del discurso que emana de los mismos confrontado a las reacciones que estos provocan en los alumnos, estos últimos se abocan a la

\footnotetext{
3 Cuento de Emilia Pardo Bazán (1851-1921).
} 
búsqueda de un lenguaje propio para expresarse en un nuevo texto creativo -poético o en prosa- elaborado individualmente, o en base a consenso, en pequeños grupos. Las autoevaluaciones junto a los trabajos producidos durante el semestre muestran que, reaprendido el procedimiento de la lectoescritura y fortalecida su confianza en sus capacidades creativas y en su potencial para producir cambios, los alumnos adquieren la seguridad imprescindible para proponer, transformar e innovar, destrezas que se pueden trasladar del ámbito académico al social. Confrontando ideas para llegar a un consenso y creando colectivamente, cada individuo enriquece su propia escritura al incorporar otros mundos y otras formas de expresión a su universo en un proceso de retroalimentación permanente.

\section{Conclusión}

\section{Del aula a la sociedad}

En conclusión, en palabras de los estudiantes, podemos inferir que esta nueva aproximación a la enseñanza logró cimentar para qué enseñamos al:

1. romper estereotipos, despertar interés, placer y amor por el estudio y la lectoescritura, y llevar a los alumnos a descubrir capacidades que desconocían:

Esperaba que esta sería una clase donde estudiaríamos la gramática de un libro y haríamos ejercicios, y honestamente pensaba que no me iba a gustar. Pero luego la clase se enfocó en hacernos estudiar la gramática a partir de la poesía y la discusión de temas sociales actuales y con ello vino la segunda sorpresa, que me gustara la poesía. (K. R. "La sorpresa")

La clase despertó en mí la pasión por escribir. Aprendí cómo convertir mis pensamientos, creencias y deseos en un mensaje inspirador que todos puedan entender. (0. A. "El poder de la escritura")

2. Producir la conjunción del placer por el estudio con el reconocimiento de la importancia de desarrollar el pensamiento crítico, no solo para mejorar su desempeño académico, sino para su incorporación a la vida social como agente de cambio.

La manera didáctica en la que logramos incluir la práctica de gramática, un tema visto como tedioso por muchos por la naturaleza tan técnica 
del mismo, en actividades creativas de escritura hizo el proceso de aprendizaje más fluido e interesante... A través de la enseñanza y práctica de habilidades de razonamiento y análisis del significado de citas, poemas y canciones más allá de las palabras escritas en la hoja de papel, la clase amplió mi mente y me proporcionó las herramientas necesarias para hacer mis propias interpretaciones sobre problemas de desigualdad e injusticia... Aprendí a favorecer un vocabulario más rico y complejo sobre las palabras simples y sobreusadas... ahora puedo expresarme muy fácilmente a través de la poesía. Anteriormente, solo podía expresarme a través de ensayos, comentarios, o presentaciones ... Este método de enseñanza ayudó a incrementar mi conocimiento de los problemas en el extranjero, así como de aquellos que afectaron a los de las generaciones anteriores lo que me ayuda a ser un individuo más completo. (D.F. “Escribiendo para el cambio”)

Más que una enseñanza, diría que el regalo más especial que me llevo de esta clase es la inspiración para trabajar y aportar mi granito de arena en la creación de un mundo igual, un mundo donde los que tenemos voz la alzamos por los que la perdieron, un mundo donde los que tenemos piernas marchamos por los que no pueden caminar, un mundo donde la preocupación y el amor por el prójimo son valores que se enseñan en el salón de clase y en la escuela. (V.S. "Más allá de los acentos")

[la clase] me retó a usar mi creatividad; asimismo aprendí a trabajar en equipo y a comprender y respetar las perspectivas de otros, y por último, me abrió mis ojos, mis oídos y mi mente a las circunstancias domésticas y extranjeras que se viven [y] me inspiró a salir de nuestra burbuja y luchar por un cambio que puede empezar con solo alzar nuestras voces por los sin voz y no tener miedo de expresar lo que sentimos. (K. A. "Abre tus ojos, tus oídos y tu mente")

Escuchar música y leer textos, citas, y poemas sobre problemas sociales ayudaron a mejorar mis habilidades de pensamiento crítico al leer y a pensar más profundamente sobre problemas diferentes en el mundo... Escribir breves ensayos y poemas con mis compañeros de clase sobre 
problemas sociales me ayudó a involucrarme en el activismo... El tema 'escribir para el cambio' me hizo pensar más en lo que puedo hacer para cambiar el mundo. (A. S. "Tres beneficios mayores")

Me encantó el concepto de practicar la gramática a través de la escritura de poemas cada clase... Las lecciones de este curso han avanzado mi comprensión del mundo y también de la gramática... Cuando nosotros analizamos sea textos o la realidad y escribimos para un cambio, estamos trabajando juntos para encender ese cambio" ... "El cambio no ha sido solamente para crear cambios sociales sino en mi manera de observar, analizar y expresar el mundo... (K. L. "El mar del entendimiento")

3. Romper el silencio, descubrir el poder de la palabra, liberarse del miedo y atreverse a opinar porque su opinión fue respetada:

Esta clase me hizo darme cuenta de que mi opinión y mi voz son importantes y de que yo sí tengo el poder de lograr cambios... Estuve feliz de poder liberar mi mente y poder poner mis pensamientos, sentimientos y opiniones en palabras. Pude expresarme de una manera diferente y aprendí lo importante que son las palabras y el impacto que pueden tener. (K. A. "Abre tus ojos, tus oídos y tu mente")

La clase nos animó a cuestionar, reflexionar y al final crear nuestro propio pensamiento independiente. No sólo me ayudó en la comprensión y en la redacción, sino a pensar en las cosas de manera más abstracta. Otro beneficio de la clase fue el aprender a crear y defender tu propia opinión. (C. S. "Escribiendo para el cambio")

Los trabajos de los alumnos y su autoevaluación personal de su desempeño en el semestre, muestran igualmente que adquirieron las herramientas para reflexionar, sintetizar, organizar, hacer conexiones, crear y comunicar sus ideas, de manera que otros puedan nutrirse y crear a su vez, superando así, los tres obstáculos que mencionamos en un comienzo había que franquear: la falta de preparación y motivación, el estereotipo de la superioridad de las disciplinas científicas sobre las humanísticas y la regla tácita del silencio. Lograron, sin duda, pensar críticamente; expresar su opinión en forma lógica y organizada oralmente y por escrito, trabajar en grupo criticando y compartiendo opiniones, analizar en profundidad y establecer 
conexiones entre diferentes tipos de lenguajes, así como entre estos y su realidad personal inmediata, aplicar las destrezas aprendidas en diferentes contextos $y_{\text {, algo }}$ muy especial, escribir creativamente haciendo uso de uno de los lenguajes más difíciles de dominar por representar la síntesis de la palabra, la emoción y el pensamiento, el lenguaje de la poesía:

\section{Una nación de verdes}

Opeposi Adetunji

Verde, blanco, verde

color de selvas y árboles

de frutas y vegetales.

color vibrante y rico

que representa a esta nación de la cual hablo

la bandera, los jerséis, los estandartes del orgullo

todo en mi país muestra esto, mas sin embargo...

los ríos están contaminados con petróleo crudo

los bosques, debido a esto, están desnudos

los pobres nunca verán el dinero verde

y la corrupción los mantiene de esta manera siempre.

Nigeria,

una nación verde, blanco, verde

donde la lucha entre

verde y blanco

rico y pobre,

fértil y estéril

permanece.

\section{La República Dominicana ${ }^{4}$}

\section{Diana Francisco}

Vengo de un país tan hermoso

que la gente pelea por la tierra

roba sus valiosos recursos

y mata para poder gobernarlo.

$4 \quad$ Publicado en The Monmouth Review, Spring 2019, p.99 
De lectores pasivos y sin, o con poco interés, y con poca capacidad de lectura, con limitadas capacidades de análisis crítico y expresión del pensamiento los alumnos pasaron a ser escritores reflexivos, activos y comprometidos. Y por si todos estos logros fueran pocos, recuperaron la pasión por la lectura, la escritura y el aprendizaje.

¿Cómo lograr que los resultados de experiencias como estas se multipliquen para romper con esa lectura uniformada, sea por intereses económicos y de producción o por ansias de poder político que nos amenaza? La pregunta queda abierta como tema para un futuro trabajo.

\section{Referencias bibliográficas}

Carnevale, A., Fasules, M., Quinn, M. \& Peltier-Campbell, K. (2019). Born to win, schooled to lose: why equally talented students don't get equal chances to be all they can be. Washington, DC, USA: Georgetown University Center on Education and the Workforce.

Church, M., Morrison, K. \& Ritchhart, R. (2011). Making Thinking Visible. USA: Jossey-Bass.

Freire, P. (2008). Cartas a quien pretende enseñar. Buenos Aires, Argentina: Siglo XXI.

Gac-Artigas, G. y Gac-Artigas, P. (1999). Directo al grano: a complete reference manual for spanish grammar. Nueva Jersey, USA: Prentice Hall, College Division.

Gac-Artigas, G. y Gac-Artigas, P. (1999). Sans détour: a complete reference manual for french grammar. Nueva Jersey, USA: Prentice Hall, College Division.

Gac-Artigas, G. y Gac-Artigas, P. (2009). iA la perfección! Para dominar la mecánica de la escritura (texto y cuaderno de ejercicios). Nueva Jersey, USA: Academic Press

Nathan, L. (2018). Reflections from Chile. Recuperado de: www.lindanathan.com

\section{Bibliografía consultada}

Belmi, P., Neale, M. A., Reiff, D. \& Ulfe, R. (2019, may 20). The social advantage of miscalibrated individuals: the relationship between social class and overconfidence and its implications for class-based inequality. Journal of Personality and Social Psychology [Advance online publication]. doi: http://dx.doi.org/10.1037/ pspi0000187

Eco, U. (2015). Número Cero (Trad. Helena Lozano). Barcelona, España: Lumen. 
Giroux, H. (2019). La guerra del neoliberalismo contra la educación superior. Barcelona: Herder Editorial.

Leslie, I. (2016, oct-nov.). The scientists who make apps addictive. Magazine The Economist. Recuperado de: https://www.1843magazine.com/features/ the-scientists-who-make-apps-addictive

Penguin Random House. (2013). Bertelsmann and Pearson complete merger to form Penguin Random House [News]. Recuperado de: https://www.bertelsmann.com/ news-and-media/news/bertelsmann-and-pearson-complete-merger-to-formpenguin-random-house.jsp

Penguin Random House. (7 de octubre de 2015). Grupo editorial crea "Cursiva", una escuela de escritura y oficios de la edición [Noticias]. Recuperado de: www. penguinrandomhouse.com

Redacción Tecnología. (2016, mayo 6). Amazon, la compañía de Jeff Bezos, planea abrir entre 300 y 400 librerías físicas por todo el territorio de los EEUU. Informativo El Diario [En línea]. Recuperado de: https://www.eldiario.es/tecnologia/dia-amazon-hizo-librero_0_480502082.html

Vass, E. (2008). New technology and habits of mind. Beyond Current Horizons. Recuperado de: https://www.researchgate.net/publication/241601789_New _technology_and_habits_of_mind

Verdú, D. (11 de julio de 2016). El gusto en la era del algoritmo, Babelia. Diario El País. Recuperado de: https://elpais.com/cultura/2016/07/07/ babelia/1467898058_835206.html

Vila-Matas, E. (2 de diciembre de 2015). El Futuro, discurso íntegro del escritor Enrique Vila-Matas en la feria del libro de México. Diario El País. Recuperado de: https:// elpais.com/cultura/2015/11/28/actualidad/1448737560_314943.html 